\title{
Erratum to: Toward a better scientific collaboration success prediction model through the feature space expansion
}

\author{
Fahimeh Ghasemian $^{1}$ - Kamran Zamanifar ${ }^{1} \cdot$ Nasser Ghasem-Aghaee $^{1} \cdot$ \\ Noshir Contractor ${ }^{2}$
}

\section{Erratum to: Scientometrics (2016) 108:777-801 \\ DOI 10.1007/s11192-016-1999-x}

In the original publication of the article, the third author family name and the affiliations of the first, second and third authors are mentioned incorrectly. The correct author name and affiliation are provided in this erratum.

The online version of the original article can be found under doi:10.1007/s11192-016-1999-x.

$\triangle$ Kamran Zamanifar

zamanifar@eng.ui.ac.ir

Fahimeh Ghasemian

ghasemianfahime@gmail.com

Nasser Ghasem-Aghaee

aghaee@eng.ui.ac.ir

Noshir Contractor

nosh@northwestern.edu

1 Department of Software Engineering, Faculty of Computer Engineering,

University of Isfahan, Isfahan, Iran

2 SONIC Lab, Northwestern University, Evanston, IL, USA 